\title{
Targeted Influential Nodes Selection in Location-Aware Social Networks
}

\author{
Susu Yang, Hui Li $(\mathbb{D}$, and Zhongyuan Jiang \\ School of Cyber Engineering, Xidian University, Xian 710126, China \\ Correspondence should be addressed to Hui Li; hli@xidian.edu.cn
}

Received 2 September 2018; Accepted 10 October 2018; Published 1 November 2018

Guest Editor: Jianxin Li

Copyright (C) 2018 Susu Yang et al. This is an open access article distributed under the Creative Commons Attribution License, which permits unrestricted use, distribution, and reproduction in any medium, provided the original work is properly cited.

Given a target area and a location-aware social network, the location-aware influence maximization problem aims to find a set of seed users such that the information spread from these users will reach the most users within the target area. We show that the problem is NP-hard and present an approximate algorithm framework, namely, TarIM-SF, which leverages on a popular sampling method as well as spatial filtering model working on arbitrary polygons. Besides, for the large-scale network we also present a coarsening strategy to further improve the efficiency. We theoretically show that our approximate algorithm can provide a guarantee on the seed quality. Experimental study over three real-world social networks verified the seed quality of our framework, and the coarsening-based algorithm can provide superior efficiency.

\section{Introduction}

In recent years, social networks have become prevalent platforms for the spread of product adoption, ideas, and news. Under this trend, influence maximization (IM) problem is becoming popular, which aims to seek $k$ users (referred to as seeds) to maximize the number of influenced users (referred to as influence spread) in the network. Kempe et al. [1] proved that this problem is NP-hard and presented an $(1-1 / e)$-approximate algorithm by greedily selecting $k$ seed users which has the maximum marginal gain of influence spread. Motivated by their work, a vast amount of studies then focused on improving the effect of influence spread and the efficiency, such as the heuristic-based algorithm PMIA [2] and the sketch-based algorithm IMM [3].

However, many real-world applications such as locationbased word-of-mouth marketing recently have locationaware requirements in IM. In [4], Li et al. focused on a location-aware IM (LAIM) query, which aims to seek $k$ users to maximize the expected influence in a query region. They assumed that the network and the location of users are given beforehand, where an index can be constructed offline, and the target region is submitted online as a query. However, such assumption may not be always satisfied as the network and IM request are given at the same time, which is exactly the scenario discussed in traditional IM works $[2,5,6]$. Besides, existing works in LAIM can only answer the problem towards simple regions such as a rectangle or a circle. However, locations of users are always complicatedly visualized and managed via maps, the atomic regions of which are not necessarily rectangles or circles. Instead, they always show up as various and complex polygons. Therefore, it is meaningful to find an efficient method to address the LAIM problem, by targeting at an arbitrary polygon region, from scratch. Below we provide a running example to elaborate this point.

Example. A company wants to sell a new product in a city. It is obvious that people in this city are potential buyers. In order to propagate this product to the public, we need to find several individuals who are the most influential in the network, and hope that, through their propagation, as many people as possible could know this product and then further purchase it.

In our paper, we present a novel algorithm framework, namely, TarIM-SF (Targeted Influence Maximization with Spatial Filtering), to deal with this problem. Given a locationaware social network and a target region, we firstly adopt a spatial filtering model (SFM) to identify the targeted users. Then we will utilize the elegant sampling approach in latest IM solutions [3] to find seed nodes. In order to further 
improve the efficiency, we have coarsened the social network. In all, our contributions in this work are as follows:

(i) We relax the target region in LAIM to arbitrary polygons, which is more practical in real applications.

(ii) Secondly, our model can address LAIM from scratch without any assumption for offline processing.

(iii) To the best of our knowledge, we are the first to prove the hardness of LAIM theoretically in detail, and for the large-scale and complex networks, we propose a coarsening-based model that can further improve the efficiency with guaranteed seed quality.

Experiments on real-world datasets Gowalla, Tweets, and Weibo demonstrate that our framework could generate a seed set with theoretically guaranteed quality, which outperforms a series of baseline methods in terms of influence spread quality. Besides, the coarsening-based algorithm can provide superior efficiency.

The rest of paper is organized as follows. Section 2 lists the related studies. Section 3 gives the definition of LAIM with proving its hardness and presents some fundamental knowledge. Afterwards, we discuss the proposed algorithm framework in Section 4. Section 5 shows the theoretical guarantee of seed quality. Section 6 reports the experimental results and some discussion. In Section 7, we conclude the paper.

\section{Related Works}

Kempe et al. [1] first formulated the influence maximization problem and proved that it is NP-hard in general, but can be approximated with $(1-1 / e-\epsilon)$ factor. They presented a greedy algorithm with a provable approximation guarantee to solve this problem. However, the greedy algorithm needs to perform the Monte Carlo simulation [7] to obtain the approximate ratio, which has a large time overhead. Furthermore, in order to improve the efficiency and the effect, there has been a large body of research works that can be divided into three types. Simulation-based methods accurately estimate influence by simulating the diffusion process repeatedly with a theoretical guarantee. Leskovec et al. [8] proposed a CELF method with the lazy-forward heuristic, which is originally designed to optimize submodular functions in [9], as well as [10-14]. Heuristic-based methods are developed to avoid using Monte Carlo simulation at the expense of solution quality. For example, Chen et al. [2] proposed to use local directed acyclic graphs to approximate the influence regions of nodes, while [15] restricting the spread of influence into communities and [6] approximating the influence spread using linear systems. Sketch-based methods resolved the inefficiency of Monte Carlo simulations without loss of accurate guarantees. Borges et al. [16] presented a nearly optimal time algorithm for IM under IC model. This method relies on reverse simulations of the diffusion process and builds sketches to estimate the influence function efficiently. In subsequential works, techniques for bounding the sketches' size are developed $[3,17-21]$ and $[3,18,19]$ are the representative ones that exhibit higher efficiency in all sketch-based methods. Moreover, Liu et al. [22] construct a community-level influence analysis model, instead of focusing on individual-influence, while [23] defining the outer influence of a community and aiming to find the most influential communities, as well as [24] constructing an influential propagation model considering the temporal-interaction between users in the social network.

Recently, more additional demands for IM problem emerged, such as considering the interests of users $[25,26]$, geographical factor, or some factors of time. Especially in order to meet the location-aware requirements in IM, Li et al. [4] proposed a method to solve location-aware IM, which works by seeking $k$ users to maximize the expected influence spread of the query region. Wang et al. [27] considered the distance between two users and defined the distance-aware IM problem. They proposed a priority based algorithm with $(1-1 / e)$-approximation ratio. The authors in [28] also studied the DAIM problem, considering the distance between the locations and the users. Zhu et al. [29] proposed Gaussian based and distance based mobility models, to derive the location-aware propagation probability in LBSN. Zhou et al. [30] take users' historical mobility behaviour into account and study the IM problem under O2O model. Li et al. [31] aim to find several seed users to maximize geographic spanning regions (MGSR) in the query region, while Li et al. [32] assume that users have their location preference and solve the IM problem for the targeted users. Furthermore, some works focus on spatial-temporal IM problem $[33,34]$, which aims to find $k$ best trajectories to be attached with an advertisement and maximizes the number of influenced users. Besides the location, the interests/topics of users are also taken into consideration in IM, and [35] proposed an algorithm that returns top- $k$ topics related to the query of a user. Su et al. [36] take not only users' interests but also their preference for locations into account, to find the targeted users, and then seek seeds to maximize the influence for targeted users.

\section{Problem Statements and Preliminaries}

\subsection{Problem Definition}

Definition 1 (LAIM). Given a location-aware social network $G=(V, E)$ where each node $v \in V$ is associated with a location (denoted as $v . \phi$ ), a budget $k$, and a target region $Q$, the location-aware influence maximization (LAIM) aims to find $k$ seed nodes (denoted as $S$ ) from $V$, such that the influence spread from $S$ can reach the most number of nodes in $Q$.

We show the hardness of LAIM problem under Independence Cascade (IC) model, which is one of the most popular diffusion models [1]. Before that, we first define a problem called Subset Cover, which will be utilized in the following content.

Definition 2 (subset cover). There are an element set $U=$ $\left\{u_{1}, u_{2}, \ldots, u_{n}\right\}$, a subset $U_{q}=\left\{u_{q 1}, u_{q 2}, \ldots, u_{q t}\right\}$ of $U$, and a collection of subsets $S=\left\{S_{1}, S_{2}, \ldots, S_{m}\right\}$ of $U$, and we wish to know whether there exist $k$ subsets in $S$, whose union is equal to $U_{q}$.

Theorem 3. The location-aware influence maximization problem is NP-hard for IC model. 

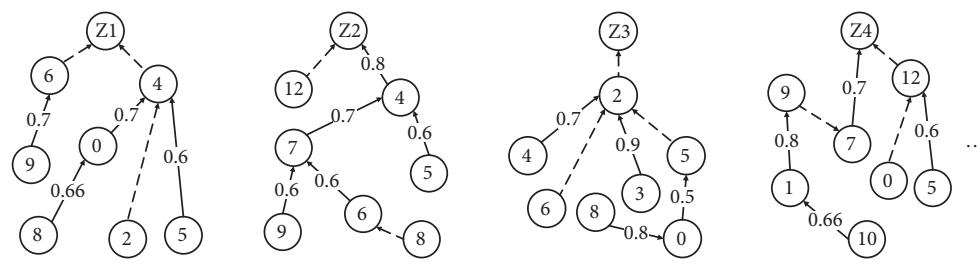

FIGURE 1: Several hypergraphs constructed from $g^{\prime}$ (dotted line represents there is a path from a node to another and the path passes through other nodes; real line represents a directed edge).

Proof. From [1], we know that the influence maximization is NP-hard by reduction from Set Cover. Here we can prove that Subset Cover problem above is also NP-hard by reduction from Set Cover problem; the process is as follows.

For $S$ and $U$ in Set Cover problem, we get a subset $U_{q}=\left\{u_{q 1}, u_{q 2}, \ldots, u_{q t}\right\}$ from $U$, and we get a new set $U^{\prime}=\left\{u_{1}, u_{2}, \ldots, u_{n}, u_{n+1}, u_{n+2}, \ldots, u_{n+t}\right\}$ by adding $t$ different elements to $U$, while getting a new collection $S^{\prime}=$ $\left\{S_{1}^{\prime}, S_{2}^{\prime}, \ldots, S_{m}^{\prime}\right\}$ of $V$ corresponding $S$, where $S_{i}^{\prime}=S_{i} \cup\left\{u_{n+j} \mid\right.$ if $\left.u_{q j} \in S_{i}, \forall j \in[t]\right\}$. This process can be completed in polynomial time. When we find a solution $A$ for Set Cover problem, the corresponding subsets $A^{\prime}$ in $S^{\prime}$ can cover all nodes in $U^{\prime}$; and if we find the solution of Subset Cover problem, Set Cover problem can also be solved. Based on this, we are able to construct a corresponding directed graph with $(m+t)$ nodes like the proof in [1]: there are node $i$ corresponding to each $S_{i}$ in $S$, node $j$ corresponding to each element $u_{q j}$ in $U_{q}$, and a directed edge $(i, j)$ with activity $p_{i j}$, where $p_{i j}=1$ if $u_{q j} \in S_{i}$; otherwise it is equal to 0 . The Subset Cover problem is equivalent to deciding if there is a set $A$ in this graph with $\sigma_{A} \geq(t+k)$, where $\sigma_{A}$ denotes the influence spread of node set $A$. Initially, if we find a set $A$ which makes $\sigma_{A} \geq(t+k)$, the Subset Cover problem will be solved, and if all $k$ nodes corresponding to sets in solution of Subset Cover are activated, all $t$ nodes corresponding to set $U_{q}$ will be activated.

3.2. Sampling Technique. Borgs et al. [16] introduced a sampling method called RIS, which first constructs a suitable number of sketches from different target nodes reversing DFS (referred to as RR sets $\mathscr{R}$ ) and then finds out $k$ users as the seed nodes with the maximum coverage of $\mathscr{R}$. The process of constructing RR sets is as follows.

Firstly, given an edge-weighted graph $G=(V, E)$, we denote $g=(V, E, p)$ as the influence graph of $G$, where $p$ is the propagation probability for edges between two user nodes. We delete every edge $e$ in $G$ with probability $\left(1-p_{e}\right)$. After that, we need to randomly choose one node in $V$ and then construct a hypergraph and get the RR set for it.

Definition 4 (RR set). Let $v$ be a node in V. A RR set for $v$ is generated by firstly sampling a graph $g^{\prime}$ from $g$ and then taking the set of nodes in $g^{\prime}$ that can reach $v$.

For instance, from hypergraphs in Figure 1, we can get the RR sets as follows: $R_{1}=\{Z 1,6,4,0,2,5,9,8, \ldots\}, R_{2}=$ $\{Z 2,12,4,7,5,9,6,8, \ldots\}, R_{3}=\{Z 3,2,6,4,3,5,0,8, \ldots\}, R_{4}=$ $\{Z 4,7,12,9,0,5,1,10, \ldots\} \cdots$. Through the construction of
$\mathscr{R}$, we can seek $k$ nodes as seeds which have the maximum coverage of $\mathscr{R}$.

\subsection{Coarsening Method}

Definition 5 ([37] coarsened influence graph). Given a social network $G=(V, E)$, let $g=(V, E, p)$ be an influence graph and $P=\left\{C_{j}\right\}_{j \in[\tau]}$ be a partition of $V$, where each $C_{j}$ is strongly connected (SC). Then, a coarsened influence graph obtained from $g$ is defined as a vertex-weighted influence graph $H=(W, F, q, w)$, where

$$
\begin{aligned}
& W=\left\{C_{j} \mid j \in[\tau]\right\}, \\
& F=\left\{\left(C_{x}, C_{y}\right) \mid C_{x} \neq C_{y}, \exists(u, v) \in E, u \in C_{x}, v\right. \\
& \left.\quad \in C_{y}\right\}, \\
& q_{C_{x} C_{y}}=1-\prod_{\substack{(u, v) \in E \\
u \in C_{x}, v \in C_{y}}}\left(1-p_{u v}\right), \quad \forall\left(C_{x}, C_{y}\right) \in F, \\
& w\left(C_{j}\right)=\left|C_{j}\right|, \quad \forall C_{j} \in W
\end{aligned}
$$

The mapping $V \longrightarrow W$ is defined as $\pi(v)=C_{j}$ such that $v \in C_{j}$. For a vertex set $S$, we let $\pi(S)=\{\pi(v) \mid v \in S\}$ and $F=\{(\pi(u), \pi(v)) \mid \pi(u) \neq \pi(v),(u, v) \in E)\}$. We follow the same coarsening process as [37]. Given an influence graph $g$, whose distribution is $D_{g}$, we first construct subgraphs $G_{1}=\left(V_{1}, E_{1}\right), \ldots G_{r}=\left(V_{r}, E_{r}\right)$, which are random graphs sampled from $D_{g}$. In these subgraphs, we identify the vertex sets connected to each other in all subgraphs, so that we get a partition $P=\left\{C_{1}, C_{2}, \ldots, C_{\tau}\right\}$ of $g$ and corresponding $H=(W, F, q, w)$ (see Figure 2).

3.4. Spatial Filtering Model. In order to figure out which nodes fall into region $Q$, the most intuitive way is to compare the location of each node with the boundary of $Q$, which is costly when $Q$ is complex or $|V|$ is very large. Herein, we will adopt an efficient method for this task. Our method works by comparing the convex hull of nodes with $Q$ and iteratively removing those nodes falling out of $Q$. Finally, we can end with a group of nodes whose convex hull is inside $Q$. In this manner, we avoid enumerating all nodes in $V$. Notably, for a point set $T$, we could use Graham Scan method [38] to seek its convex hull. Let $T=\left\{v_{1} . \phi, \ldots, v_{n} . \phi\right\}$ be the nodes' locations and $\left(q_{1}, \ldots, q_{m_{q}}\right)$ be the boundary of $Q$ as a point sequence. Then the process of finding target nodes in $Q$ is shown in Algorithm 1. 


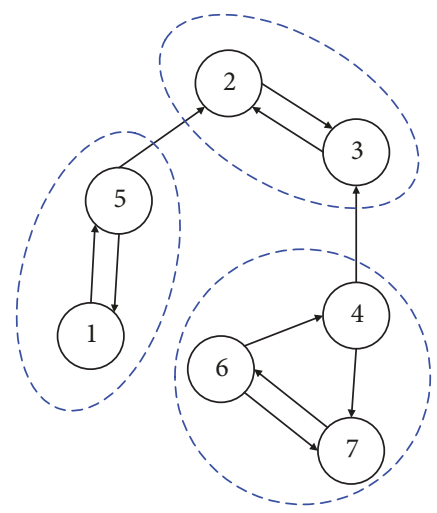

(a) Subgraph 1

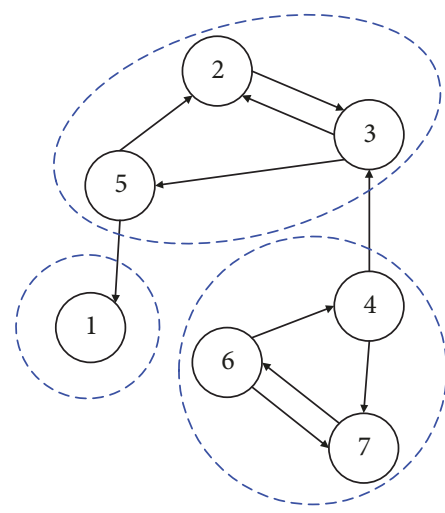

(b) Subgraph 2

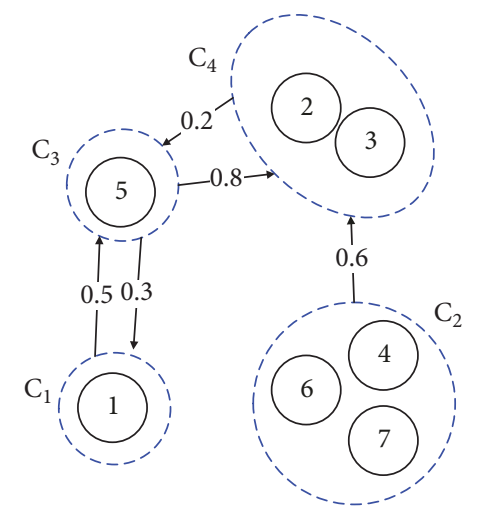

(c) Coarsened influence graph

FIgURE 2: Two subgraphs of $g$ and the coarsened influence graph $H$.

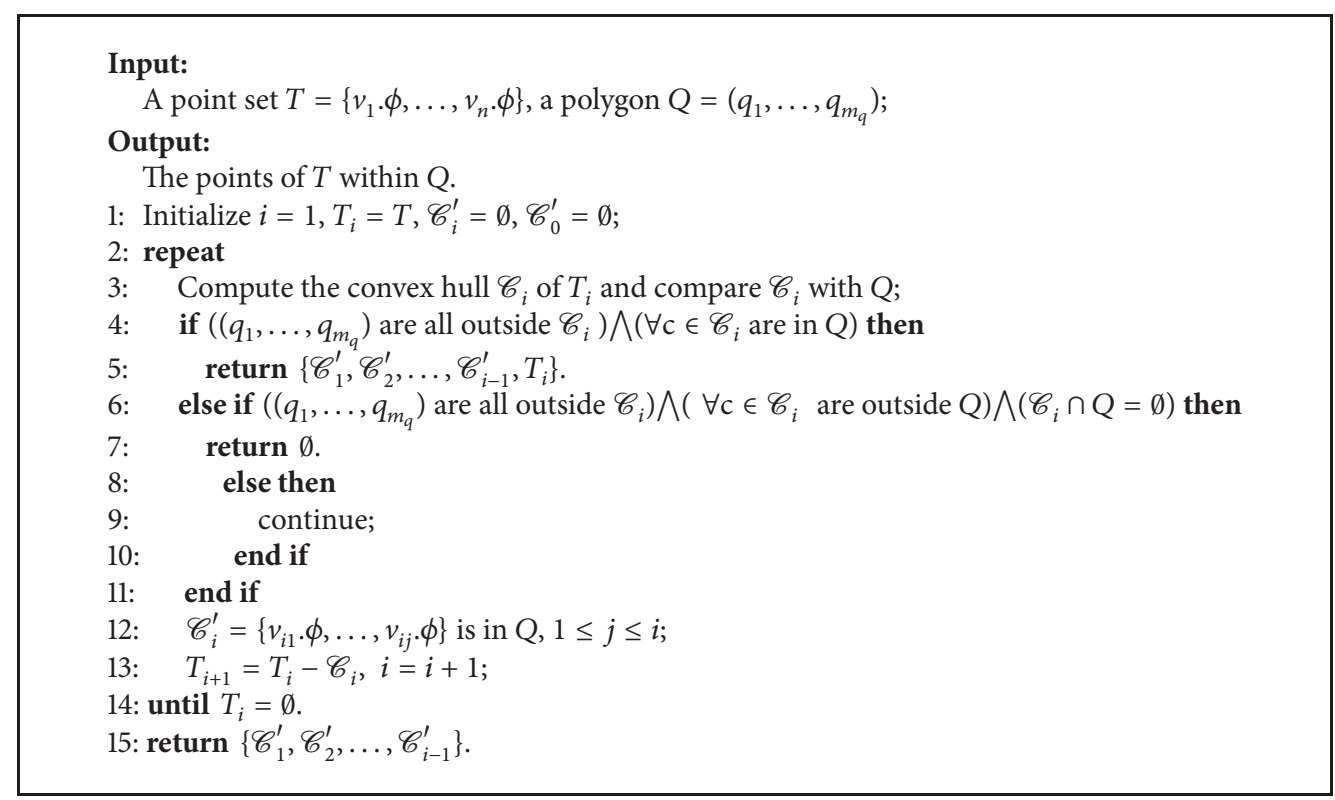

Algorithm 1: SFM $(T, Q)$.

\section{TarIM-SF Framework}

Here we describe our TarIM-SF framework in detail. In our LAIM problem, target users change from the whole network in classic IM problem into users in a target region. As the construction of RR sets starts from the target users, it is reasonable for us to construct enough $\mathrm{RR}$ sets $\mathscr{R}$ over the whole network for users in the target region within our problem and then choose the node set $s$ which maximizes the coverage of $\mathscr{R}$ (referred to as $F_{\mathscr{R}}(s)$ ). Based on this idea, we first need to identify the users in the target region before constructing RR sets, which is addressed using the method proposed in Section 3.4. Moreover, in order to improve the efficiency of constructing RR sets, we have coarsened the network using the method proposed in Section 3.3. More details are given in Algorithm 2.

For instance, in Figure 3, given a location-aware social network $G, k=1$, and a query region $Q$ (such as a triangle), we first use SFM (line 3 ) to identify the goal users $\{3,4,6\}$, then we coarsen the whole network, and we get the partition $\mathrm{P}=\left\{C_{1}, C_{2}, C_{3}, C_{4}\right\}$, where $C_{1}=\{1\}, C_{2}=$ $\{4,6,7\}, C_{3}=\{5\}, C_{4}=\{2,3\}$ (line 4). Next we will sample the coarsened influence graph and construct enough RR sets for the goal users (line 5). In the coarsened influence graph, the probability of partition node $C_{4}$ being chosen to construct RR set is $1 / 3$, and there is $2 / 3$-probability for node $C_{2}$. As a result, we get RR sets: $R_{1}=\left\{C_{2}, C_{4}\right\}, R_{2}=\left\{C_{4}\right\}, R_{3}=\left\{C_{2}, C_{4}\right\}$. We can see that node $C_{4}$ has the maximum coverage of $\mathscr{R}$, including $R_{1}, R_{2}, R_{3}$. In turn for the original network graph, we choose one user node randomly in $C_{4}$ as the seed, such as node 2 (lines 6-10).

In our framework, the first step is to identify the users in the target region $Q$, whose complexity depends on the location distribution of all nodes. In case that all users' locations are uniformly randomly distributed, the time complexity for identifying users within the target region is $O\left(n^{1 / 3} m_{q}\right)$ 


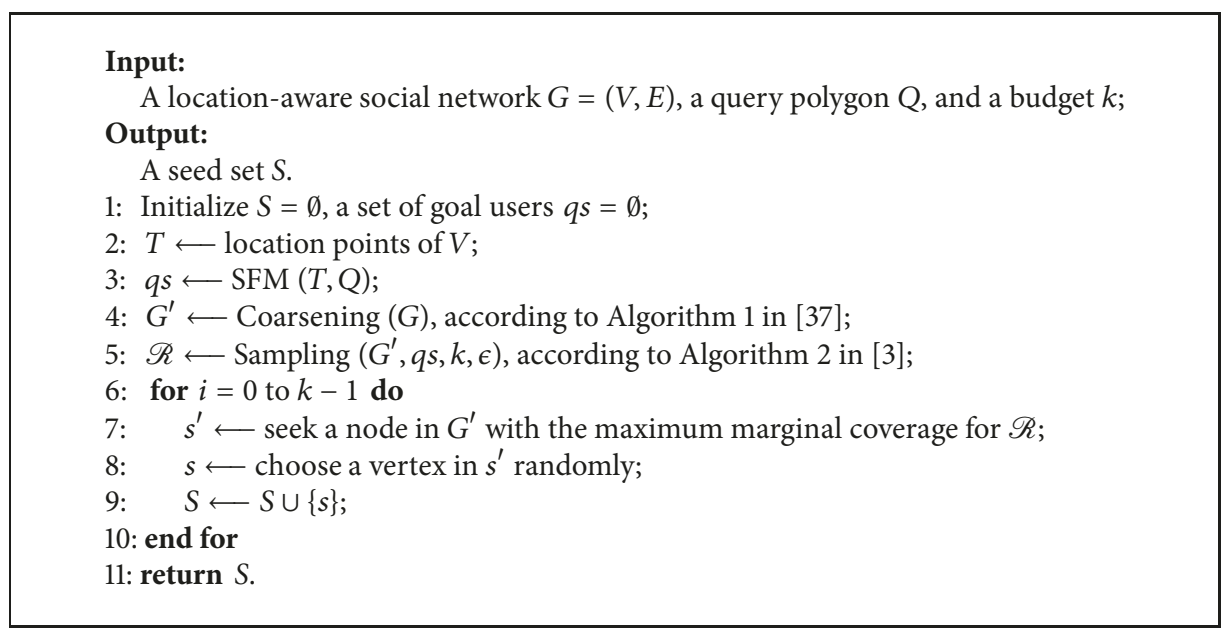

Algorithm 2: TarIM-SF $(G, Q, k, \epsilon)$.

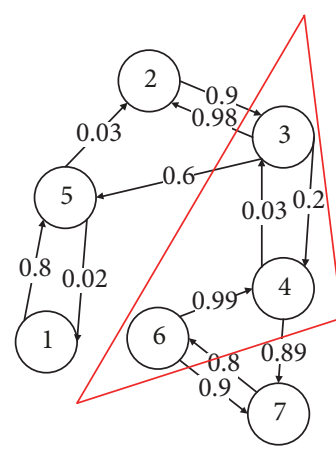

Step 1: A query

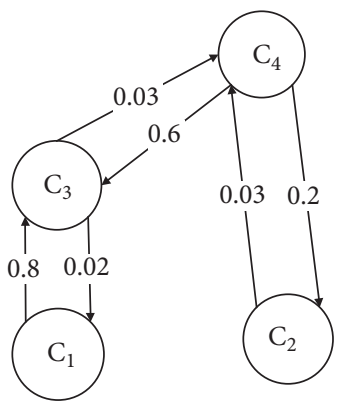

Step 2: Coarsening

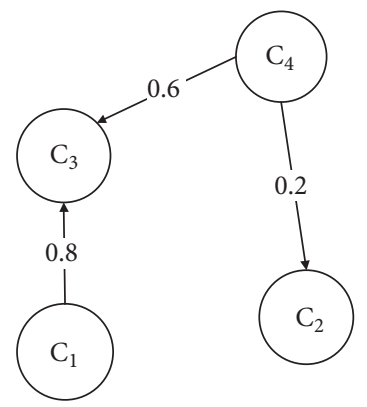

Step 3: Sampling

FIgure 3: An example.

under our spatial filtering model, where $n=|V|$ and $m_{q}$ is the number of points for $Q$. The second step is to coarsen the network, which requires $O(r(|V|+|E|))$ time, where $r$ denotes the number of random subgraphs sampled from g. Afterwards, we use algorithm in [3] to seek solution in coarsened influence network, and the time of this step is spent on constructing RR sets for target region. The complexity of this process is $O\left(\sum_{j \in[\mathscr{R}]} w\left(R_{j}\right)\right), w\left(R_{j}\right)$ denoting the edges number of the $i$-th RR set, where $|\mathscr{R}|$ is decided by the parameters $\lambda^{\prime}$ and $\lambda^{*}$ in [3], as well as the number of nodes and edges in the coarsened influence graph.

\section{Effectiveness Study}

We will subsequently conduct a theoretical study over the seed quality of our algorithm framework for both cases when coarsening is present or not.

5.1. Seed Quality without Coarsening. In [1], it has been proved that, under Independent Cascade model, the result influence function $\sigma(\cdot)$ is sunmodular. Here we define $\sigma(\cdot)$ as the target influence spread, which is the number of influenced nodes in target region. It is easy to see that, for any sets $S$ and $T, S \subseteq T$, and any elements $v, \sigma(S \cup\{v\})-\sigma(S) \geq$ $\sigma(T \cup\{v\})-\sigma(T) \geq$ also holds. Hence, the function $\sigma(\cdot)$ is submodular.

Theorem 6 (see [1]). For a nonnegative, monotone submodular function $f$, let $S_{k}^{o}$ be a size- $k$ set by selecting one element at a time, each time choosing the element which has the maximum marginal function value. Assuming $S_{k}^{*}$ is the set that maximizes the value of $f$ over all $k$-element sets, then $f\left(S_{k}^{o}\right) \geq(1-1 / e)$. $f\left(S_{k}^{*}\right)$; in other words, $S_{k}^{o}$ guarantees a $(1-1 / e)$-approximation.

So if we get a seed set $S_{\text {out }}$ by adopting a greedy algorithm, $S_{\text {out }}$ is a $(1-1 / e)$-approximate solution for the locationaware influence maximization problem. Then we will show the performance guarantee when we adopt IMM method [3] as the greedy approach.

Lemma 7 (see [16]). For any seed set $S$ and any vertex $v$, the probability that a diffusion process from $S$ can activate $v$ equals the probability that $S$ overlaps an RR set for $v$.

We generate a sizeable set $\mathscr{R}$ of random RR sets for the nodes in target region $Q$, and for any seed set $S$, the fraction $F_{\mathscr{R}}(S)$ of RR sets in $\mathscr{R}$ covered by $S$ is the unbiased estimator 
of $\mathbb{E}(\sigma(S)) / n_{q}$, where $n_{q}$ is the number of vertices in target region Q. In TIM ${ }^{+}$[17], it has been proved that the solution which covers the maximum number of RR sets provides a $(1-1 / e-\epsilon)$-approximation with at least $\left(1-1 / n^{\ell}\right)$ probability, but the number of RR sets is at least $\lambda / O P T$, where $O P T$ is the maximum expected influence of any size- $k$ nodes set in $G$ and $\lambda$ is a function of $k, \ell, n$, and $\epsilon$. In IMM, it seeks a tighter lower bound $L B$ of OPT than $\mathrm{TIM}^{+}$. Next, based on the analysis of the performance guarantee in the IMM, we will describe the parameter settings and performance guarantee in our framework.

Let $R_{1}, R_{2}, \ldots, R_{\theta}$ be the sequence of generated RR sets for nodes in the target region $Q$. Let $S_{k}$ be any size- $k$ seed set in $G$ and $x_{i}$ be random variable that equals 0 if $S_{k} \cap R_{i}=\emptyset$ and 1 otherwise; then based on Lemma 7, we have

$$
\mathbb{E}\left[\sigma\left(S_{k}\right)\right]=\frac{n_{q}}{\theta} \cdot \mathbb{E}\left[\sum_{i=1}^{\theta} x_{i}\right] .
$$

Consider $S_{k}^{*}$ is the size- $k$ node set with the maximum expected influence; let $O P T=\mathbb{E}\left[\sigma\left(S_{k}^{*}\right)\right]$. From (2), we can get that $n_{q} \cdot F_{\mathscr{R}}\left(S_{k}^{*}\right)$ is an unbiased estimator of OPT. By Corollary 2 in which we set $p=O P T / n_{q}$ and Lemma 3 in [3], we have the following.

Lemma 8. Let $\epsilon_{1}>0, \delta_{1} \in(0,1)$, and

$$
\theta_{1}=\frac{2 n_{q} \cdot \log \left(1 / \delta_{1}\right)}{O P T \cdot \epsilon_{1}^{2}}
$$

if $\theta \geq \theta_{1}$, then $n_{q} \cdot F_{\mathscr{R}}\left(S_{k}^{*}\right) \geq\left(1-\epsilon_{1}\right) \cdot$ OPT holds with at least $\left(1-\delta_{1}\right)$ probability.

Assuming that our framework without coarsening returns a solution $S_{k}^{\prime}$, and if $n_{q} \cdot F_{\mathscr{R}}\left(S_{k}^{*}\right) \geq\left(1-\epsilon_{1}\right) \cdot O P T$ holds, according to the properties of the greedy approach, then

$$
\begin{aligned}
n_{q} \cdot F_{\mathscr{R}}\left(S_{k}^{\prime}\right) & \geq\left(1-\frac{1}{e}\right) \cdot n_{q} \cdot F_{\mathscr{R}}\left(S_{k}^{*}\right) \\
& \geq\left(1-\frac{1}{e}\right) \cdot\left(1-\epsilon_{1}\right) \cdot O P T
\end{aligned}
$$

Lemma 9. Let $\epsilon_{1}<\epsilon, \delta_{2} \in(0,1)$, and

$$
\theta_{2}=\frac{(2-2 / e) \cdot n_{q} \cdot \log \left(\left(\begin{array}{l}
n \\
k
\end{array}\right) / \delta_{2}\right)}{O P T \cdot\left(\epsilon-(1-1 / e) \cdot \epsilon_{1}\right)^{2}},
$$

if (4) holds and $\theta \geq \theta_{2}$, then $\mathbb{E}\left(\sigma\left(S_{k}^{\prime}\right)\right) \geq(1-1 / e-\epsilon) \cdot \mathrm{OPT}$ holds with at least $\left(1-\delta_{2}\right)$ probability.

Based on Lemmas 8 and 9, we have the following.

Theorem 10. Given any $\epsilon_{1} \leq \epsilon$ and any $\delta_{1}, \delta_{2} \in(0,1)$ with $\delta_{1}+$ $\delta_{2} \leq 1 / n^{\ell}$, if $\theta \geq \max \left\{\theta_{1}, \theta_{2}\right\}$, the result which IMM returns is $a(1-1 / e-\epsilon)$-approximate solution with $\left(1-1 / n^{\ell}\right)$ probability.
For the parameters in Theorem 10, we set $\delta_{1}=\delta_{2}=$ $1 /\left(2 n^{\ell}\right)$, and under this setting, $\theta$ is minimized when $\theta_{1}=\theta_{2}$, and $\epsilon_{1}=\epsilon \cdot \alpha /((1-1 / e) \cdot \alpha \cdot \beta)$, where

$$
\begin{aligned}
& \alpha=\sqrt{\ell \log n+\log 2}, \\
& \beta=\sqrt{\left(1-\frac{1}{e}\right) \cdot\left(\log \left(\begin{array}{l}
n \\
k
\end{array}\right)+\ell \log n+\log 2\right)} .
\end{aligned}
$$

In this case, $\theta=\left(2 n_{q} \cdot((1-1 / e) \cdot \alpha+\beta)^{2}\right) /\left(O P T \cdot \epsilon^{2}\right)$, and conversely if we set $\theta=\lambda^{*} / O P T$, where

$$
\lambda^{*}=\frac{2 n_{q} \cdot((1-1 / e) \cdot \alpha+\beta)^{2}}{\epsilon^{2}},
$$

the seed set $S_{k}^{\prime}$ that covers the maximum number of RR sets is a $(1-1 / e-\epsilon)$-approximation. However, as $O P T$ is unknown in advance, we will find a tight lower bound $L B$ of $O P T$ as IMM method. In the sampling phase in IMM, Lemma 6, Lemma 7, and Lemma 8 in [3] proved that $L B \leq O P T$ and $L B$ is close to $O P T$. And based on that, we can also prove that $L B \leq O P T$ and $L B$ is a tight lower bound of $O P T$ with a high probability by changing $n$ to $n_{q}$.

Theorem 11. With at least $\left(1-1 / n^{\ell}\right)$ probability, sampling algorithm in our framework returns a set $\mathscr{R}$ of $R R$ sets with $|\mathscr{R}| \geq \lambda^{*} / O P T$, where $\lambda^{*}$ is as defined in (7).

Combining Theorem 10 and Theorem 11, our algorithm framework without coarsening can get a solution $S_{\text {out }}$ which is a $(1-1 / e-\epsilon)$-approximation with a high probability.

5.2. Seed Quality for Coarsening Method. In this part, we will show the result seed set $S_{\text {out }}$ can achieve $(1-1 / e-\epsilon)$. $\alpha_{g}$-approximation, when coarsening technique is adopted to improve the efficiency. Based on the study in [37], for the target region $Q$, we will get the following equation:

$$
\inf _{g}(Q, S)=\sum_{E^{\prime} \subseteq E} p_{E^{\prime} \mid E} \cdot R_{\left(V, E^{\prime}\right)}(Q, S),
$$

where $p_{E^{\prime} \mid E}=\prod_{e \in E^{\prime}} p_{e} \cdot \prod_{e \in E \backslash E^{\prime}}\left(1-p_{e}\right), R_{g}(Q, S)$ is the number (sum of weights) of vertices in $Q$ that are reachable from $S$ in $g$, and $\inf _{g}(Q, S)$ is the number of vertices in $Q$ that $S$ can activate in $g$, which equals $\sigma(S)$ in Section 5.1 and is submodular. have

In the coarsened influence graph $H=(W, F, q, w)$, we also

$$
\inf _{H}(Q, \pi(S))=\sum_{Y \subseteq F} q_{Y \mid F} \cdot R_{(W, Y)}(Q, \pi(S)) .
$$

It denotes the sum of weights for nodes in $Q$ that $\pi(S)$ can activate in $H$. After coarsening the network, we define $I=$ $\left(V, E, p^{\prime}\right)$, where $p_{u v}^{\prime}=1$ if $u, v \in C_{j}$ for all $j \in[\tau]$; otherwise $p_{u v}^{\prime}=p_{u v}$, and $\inf _{I}(Q, S)$ is the number of users in target region $Q$ that $S$ can activate in $I$.

Here, we show the relationship between $H$ and $g$ in terms of influence function through $I$, which has the same structure as $g$. 


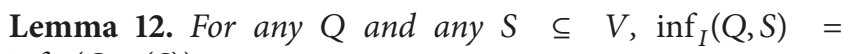
$\inf _{H}(Q, \pi(S))$.

Proof. For any $u$ and $v$ in $V$, " $u$ can reach $v$ through the edges in $E$ with $p$ " if and only if " $\pi(u)$ can reach $\pi(v)$ through the edges in $F$ with $q$ ", since every subgraph $\left(C_{j}, E_{j}\right), j \subseteq[\tau]$, is strongly connected. Therefore, it holds that $R_{(V, E)}(Q, S)=$ $R_{(W, Y)}(Q, \pi(S))$. Thus,

$$
\begin{aligned}
\inf _{I}(Q, S) & =\sum_{E^{\prime} \subseteq E} p_{E^{\prime} \mid E}^{\prime} \cdot R_{\left(V, E^{\prime}\right)}(Q, S) \\
& =\sum_{Y \subseteq F} q_{Y \mid F} \cdot R_{(W, Y)}(Q, \pi(S)) \\
& =\inf _{H}(Q, \pi(S))
\end{aligned}
$$

For $g$ and $I$, we also can find the relationship between them as follows.

Lemma 13. For any $Q$ and any $S \subseteq V$, $\inf _{g}(Q, S) \leq \inf _{I}(Q, S)$.

Proof. $g$ and $I$ are the influence graph with the same structure, but $p_{e} \leq p^{\prime}{ }_{e}$ for every edge $e$. So $\inf _{g}(Q, S) \leq \inf _{I}(Q, S)$ for any $S \subseteq V$.

For any subgraph $g\left[V^{\prime}\right]=\left(V^{\prime}, E^{\prime}\right)$ of $g, V^{\prime} \subseteq V$, its strongly connected reliability, denoted as $\operatorname{Rel}\left(g\left[V^{\prime}\right]\right)$, is defined the same as in Equation 14 in [37] and indicates the probability that $g\left[V^{\prime}\right]$ is strongly connected.

Lemma 14. $\inf _{I}(Q, S) \leq \prod_{j \in[\tau]} \operatorname{Rel}\left(g\left[C_{j}\right]\right)^{-1} \cdot \inf _{g}(Q, S)$, for any $Q$ and $S \subseteq V$.

Proof. For each $j \in[\tau]$, we define $E_{j}=\left\{(u, v) \in E \mid u, v \in C_{j}\right\}$ and $E_{0}=E \backslash\left(\bigcup_{j \in[\tau]} E_{j}\right)$. For $X_{j} \subseteq E_{j}(j \in[\tau])$ and $X_{0} \subseteq$ $E_{0}, R_{\left(V, X_{0} \cup \cup_{j} X_{j}\right)}(S)=R_{\left(V, X_{0} \cup \cup_{j} E_{j}\right)}(S)$ holds if every subgraph $\left(C_{j}, X_{j}\right)$ for $j \in[\tau]$ is strongly connected. Thus

$$
\begin{aligned}
& \inf _{g}(Q, S)=\sum_{X_{1} \subseteq E_{1}} p_{X_{1} \mid E_{1}} \cdots \sum_{X_{\tau} \subseteq E_{\tau}} p_{X_{\tau} \mid E_{\tau}} \\
& \sum_{X_{0} \subseteq E_{0}} p_{X_{0} \mid E_{0}} \cdot R_{\left(V, X_{0} \cup \cup_{j} X_{j}\right)}(S) \\
& \geq \sum_{\substack{X_{1} \subseteq E_{1} \\
\left(C_{1}, X_{1}\right) \text { is SC }}} p_{X_{1} \mid E_{1}} \cdots \sum_{\substack{X_{\tau} \subseteq E_{\tau} \\
\left(C_{\tau}, X_{\tau}\right) \text { is SC }}} p_{X_{\tau} \mid E_{\tau}} \\
& \sum_{X_{0} \subseteq E_{0}} p_{X_{0} \mid E_{0}} \cdot R_{\left(V, X_{0} \cup \cup_{j} X_{j}\right)}(S) \\
& =\prod_{j \in[\tau]} \operatorname{Rel}\left(g\left[C_{j}\right]\right) \cdot \inf _{I}(Q, S)
\end{aligned}
$$

Theorem 15. For any $Q$ and any $S \subseteq V$, inf $_{g}(Q, S) \leq \inf _{H}(Q$, $\pi(S)) \leq \prod_{j \in[\tau]} \operatorname{Rel}\left(g\left[C_{j}\right]\right)^{-1} \cdot \inf _{g}(Q, S)$.
Let $S^{*}$ and $T^{*}$ be the optimal solutions of size $k$ for $g$ and $H$, respectively. Based on Lemma 12 and Lemma 13, we can sure that $\inf _{H}\left(Q, T^{*}\right) \geq \inf _{g}\left(Q, S^{*}\right)$, and have

$$
\begin{aligned}
\inf _{H}\left(Q, S_{\text {out }}\right) & \geq\left(1-\frac{1}{e}-\epsilon\right) \cdot \inf _{H}\left(Q, T^{*}\right) \\
& \geq\left(1-\frac{1}{e}-\epsilon\right) \cdot \inf _{g}\left(Q, S^{*}\right)
\end{aligned}
$$

Then applying Lemma 14, we have

$$
\begin{aligned}
& \inf _{H}\left(Q, S_{\text {out }}\right) \\
& \quad \geq \prod_{j \in[\tau]} \operatorname{Rel}\left(g\left[C_{j}\right]\right) \cdot\left(1-\frac{1}{e}-\epsilon\right) \cdot \inf _{g}\left(Q, S^{*}\right)
\end{aligned}
$$

Therefore, our coarsening-based algorithm achieves a (1$1 / e-\epsilon) \cdot \alpha_{g}$-approximate solution for $g$, where $\alpha_{g}$ refers to $\prod_{j \in[\tau]} \operatorname{Rel}\left(g\left[C_{j}\right]\right)$.

\section{Results and Discussion}

In this section, we conduct experiments on several real-world datasets to test the performance of the proposed algorithm framework. All algorithms are implemented in $\mathrm{C}++$ and run on Ubuntu 16.10 machine with Intel Core i5-6500 quad-core, 3.20GHz, 16GB RAM.

In the following experiments, we use three location-aware social networks, namely, Gowalla, Tweets, and Weibo. The statistics for the datasets are listed in Table 1 ( $n$ represents the number of vertices and $m$ represents the number of edges). By default, we use a randomly selected $Q$ for all datasets, and the number of user nodes falling in $Q$ is denoted as $n_{q}$. We conduct our experiments on WC model, which is widely used for information diffusion. For the weight of every edge, we set the probability of an edge $(u, v)$ as $1 / N_{v}$, where $N_{v}$ denotes the in-degree of user node $v$. In TarIM-SF, we set $\epsilon=0.1$.

6.1. Comparison with Baseline. We evaluate the performance of our algorithm framework TarIM-SF compared with method Assembly in [4] under WC model. In order to estimate the performance in general, we selected three regions with fixed $n_{q}$ for each dataset $\left(n_{q} \approx 20 \mathrm{~K}\right.$ for Gowalla, $n_{q} \approx$ $120 \mathrm{~K}$ for Tweets, and $n_{q} \approx 120 \mathrm{~K}$ for Weibo) and reported the average performance, while varying $k$ for Gowalla from 10 to 50 and $k$ for Tweets and Weibo from 100 to 500 . In Figure 4, we can see that the target influence spread of seeds in our framework is obviously superior to that of Assembly, especially on Tweets and Gowalla.

6.2. Effect of Coarsening. As mentioned above, we adopt coarsening technique so as to improve the efficiency for large-scale social networks. In this part, we did a series of experiments on Weibo, a large-scale network with about a million users. In order to justify how the parameter $r$ in coarsening method will affect the target influence spread of seeds, we report the Relative Error, which measures the gap between the real influence spread of seed set we get and its 
TABLE 1: Datasets statistic.

\begin{tabular}{lcc}
\hline Datasets & $n$ & $m$ \\
\hline Gowalla & 196,585 & 351,452 \\
Tweets & 554,372 & $2,402,720$ \\
Weibo & $1,020,730$ & $16,490,916$ \\
\hline
\end{tabular}

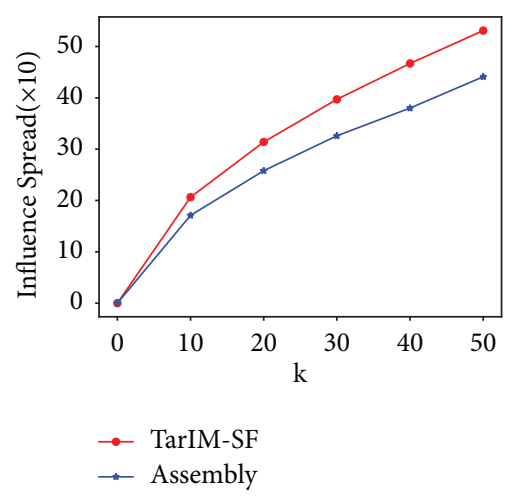

(a) $\operatorname{Gowalla}\left(n_{q} \approx 20 K\right)$

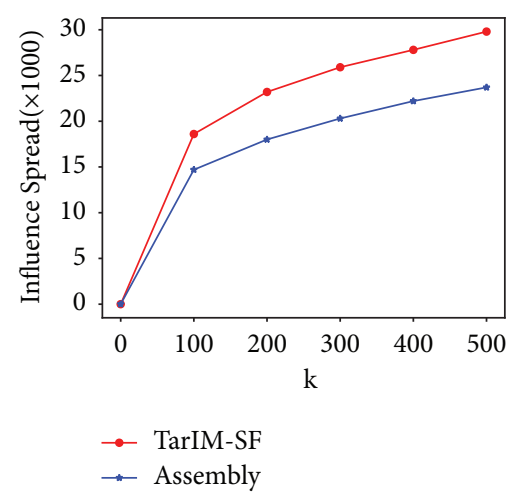

(b) $\operatorname{Tweets}\left(n_{q} \approx 120 K\right)$

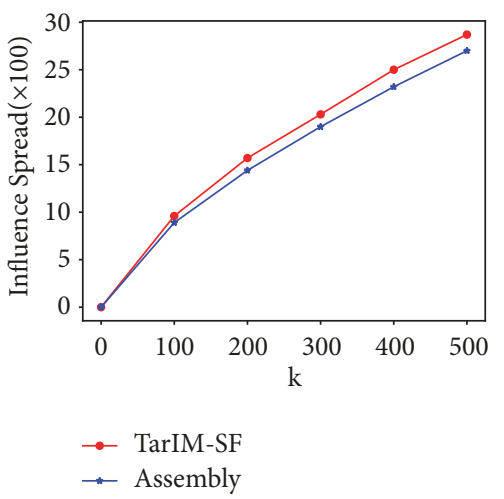

(c) $\operatorname{Weibo}\left(n_{q} \approx 120 K\right)$

FIGURE 4: Influence spread under WC model.

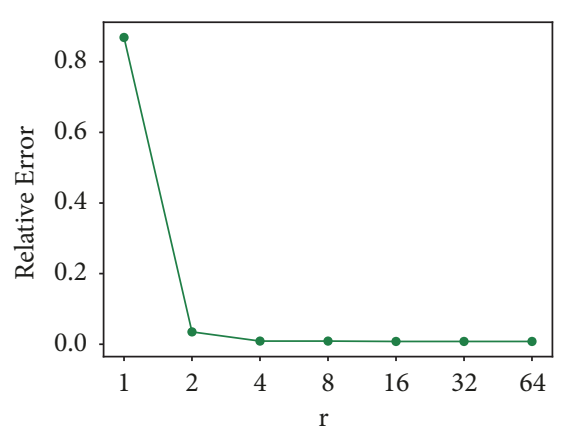

(a) Effect of $r$

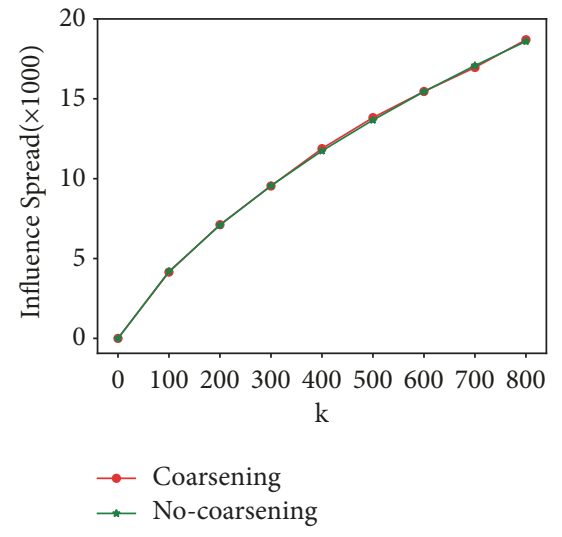

(b) Influence $\operatorname{spread}(r=16)$

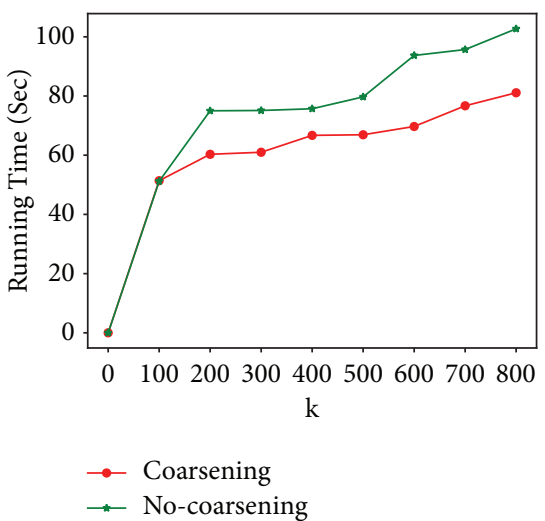

(c) Running time $(r=16)$

FIGURE 5: Effect when using coarsening.

estimated influence spread. We set $k=300$ and the target region as the whole network. Figure 5(a) shows the relative error is decreasing when $r$ becomes bigger. When $r=16$, the estimated influence spread of seeds using our algorithm with coarsening is nearly equal to the eventual influence spread of seeds without coarsening. Figures 5(b) and 5(c) indicate that the running time for coarsening approach is significantly less than that of noncoarsening one, without loss of seed quality.

6.3. Varying the Size and Shape of $Q$. We also conducted another group of experiments by varying $Q$ in terms of both size and shape. Specifically, we vary $Q$ as triangle, tetragon, and pentagon, respectively. For each shape, we vary the size at several different levels and report the performance of our algorithm (shown in Figure 6, $|Q|=n_{q}$ ). It justifies that our algorithm can work on target region with arbitrary polygon shapes. Besides, the time spent on the first phase in our algorithm framework increases slightly as the shape of $Q$ varied from pentagon to triangle at the same scale, because nodes in convex hull need to be compared with the polygon queried and the fewer the number of polygon edges, the fewer the comparison times and the less time.

\section{Conclusions}

In this study, we present a novel model that can address LAIM with a target region that can be an arbitrary polygon. Our framework uses a spatial filtering model to initially figure out the nodes falling into the target polygon. Afterwards, a coarsening process is conducted over the network. Then, the state-of-the-art sampling algorithm adopted in traditional IM is used to find the solution. We theoretically prove the 

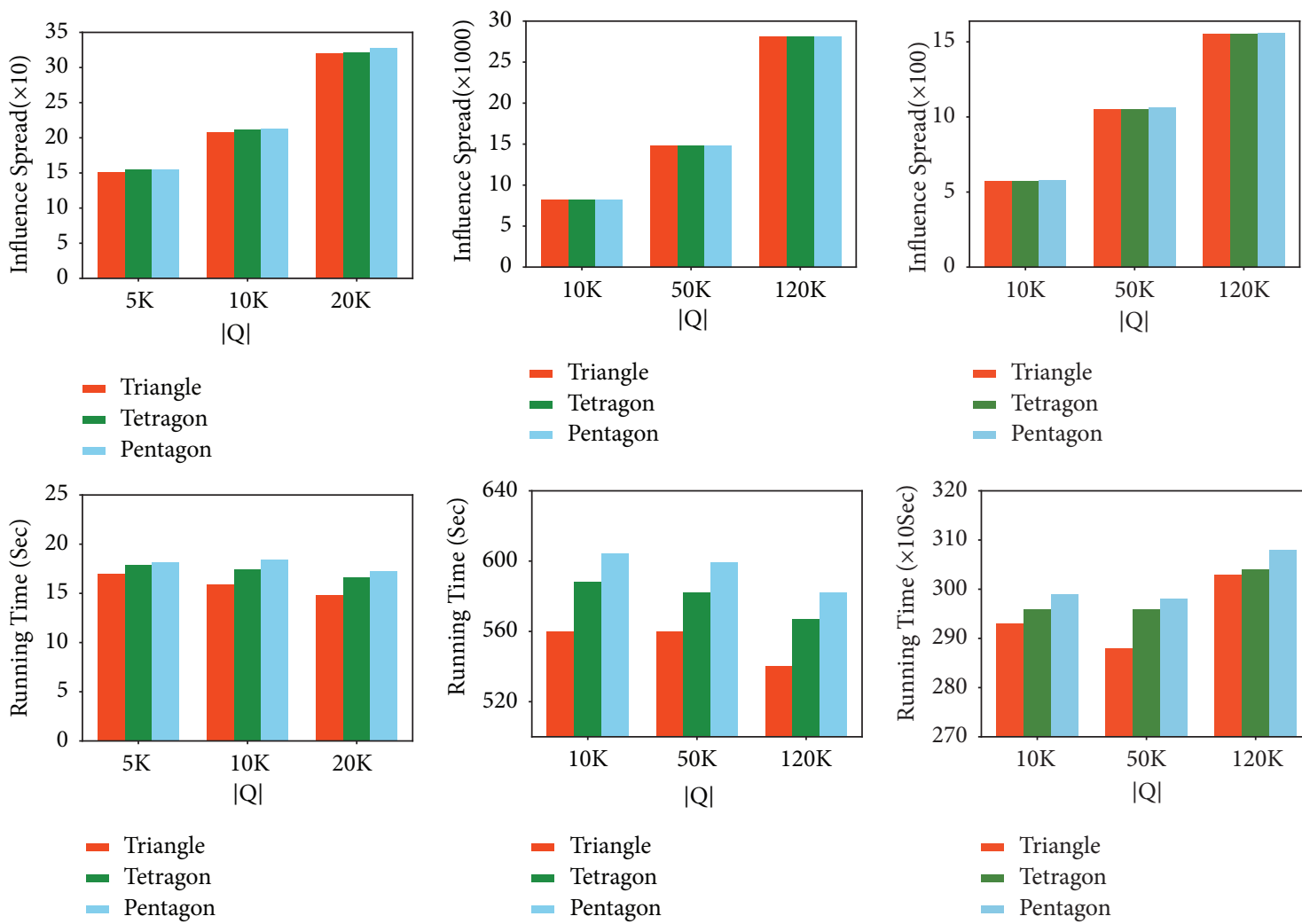

$$
\begin{aligned}
& \text { Triangle } \\
& \text { - Tetragon } \\
& \text { - Pentagon }
\end{aligned}
$$

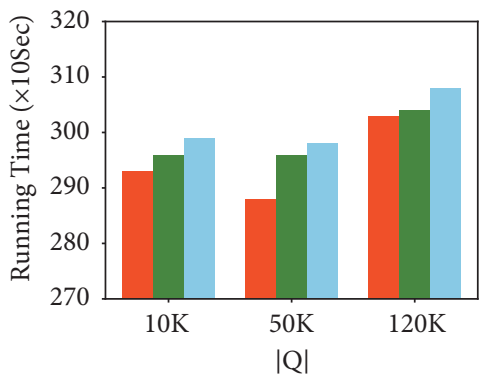

(a) $\operatorname{Gowalla}(k=30)$

(b) $\operatorname{Tweets}(k=300)$

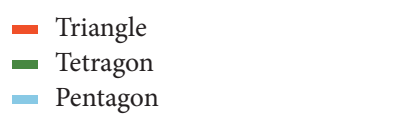

(c) $\operatorname{Weibo}(k=300)$

FIGURE 6: Influence spread and running time in terms of size and shape of $Q$.

influence spread guarantee in both noncoarsened and coarsened cases. Empirical study over three real-world datasets demonstrates that our framework outperforms the baseline algorithm in terms of influence spread and is efficient in largescale networks.

\section{Data Availability}

This study is based on the datasets Gowalla, Tweets, and Weibo provided in [4], and they are available at http:// dbgroup.cs.tsinghua.edu.cn/ligl/laim/.

\section{Conflicts of Interest}

The authors declare that there are no conflicts of interest regarding the publication of this paper.

\section{Acknowledgments}

The work is supported by the National Natural Science Foundation of China (Grant No. 61672408), Fundamental Research Funds for the Central Universities (No. JB181505), Natural Science Basic Research Plan in Shaanxi Province of China (No. 2018JM6073), and China 111 Project (No. B16037).

\section{References}

[1] D. Kempe, J. Kleinberg, and É. Tardos, "Maximizing the spread of influence through a social network," in Proceedings of the 9th ACM SIGKDD International Conference on Knowledge Discovery and Data Mining (KDD '03), pp. 137-146, New York, NY, USA, August 2003.

[2] W. Chen, C. Wang, and Y. Wang, "Scalable influence maximization for prevalent viral marketing in large-scale social networks," in Proceedings of the 16th ACM SIGKDD International Conference on Knowledge Discovery and Data Mining, KDD, pp. 1029-1038, USA, July 2010.

[3] Y. Tang, Y. Shi, and X. Xiao, "Influence maximization in nearlinear time: a martingale approach," in Proceedings of the ACM SIGMOD International Conference on Management of Data, (SIGMOD '15), pp. 1539-1554, Melbourne, Australia, June 2015.

[4] G. Li, S. Chen, J. Feng, K. Tan, and W. Li, "Efficient locationaware influence maximization," in Proceedings of the the 2014 ACM SIGMOD international conference, pp. 87-98, Snowbird, Utah, USA, June 2014.

[5] A. Goyal, W. Lu, and L. V. S. Lakshmanan, "SIMPATH: An efficient algorithm for influence maximization under the Linear Threshold model," in Proceedings of the 11th IEEE International Conference on Data Mining, ICDM 2011, pp. 211-220, Canada, December 2011.

[6] K. Jung, W. Heo, and W. Chen, "IRIE: Scalable and robust influence maximization in social networks," in Proceedings of the 12th IEEE International Conference on Data Mining, ICDM 2012, pp. 918-923, Belgium, December 2012.

[7] D. J. C. MacKay, "Introduction to monte carlo methods," Learning in Graphical Models, vol. 30, no. 90, pp. 175-204, 1998.

[8] J. Leskovec, A. Krause, C. Guestrin, C. Faloutsos, J. Vanbriesen, and N. Glance, "Cost-effective outbreak detection in networks," 
in Proceedings of the 13th ACM SIGKDD International Conference on Knowledge Discovery and Data Mining (KDD '07), pp. 420-429, New York, NY, USA, August 2007.

[9] M. Minoux, "Accelerated greedy algorithms for maximizing submodular set functions," Optimization Techniques, pp. 234243, 1978 .

[10] A. Goyal, W. Lu, and L. V. S. Lakshmanan, “CELF++: optimizing the greedy algorithm for influence maximization in social networks," in Proceedings of the 20th International Conference Companion on World Wide Web, (WWW'11), pp. 47-48, Hyderabad, India, April 2011.

[11] N. Ohsaka, T. Akiba, Y. Yoshida, and K. I. Kawarabayashi, "Fast and accurate influence maximization on large networks with pruned monte-carlo simulations," in Proceedings of the TwentyEighth AAAI Conference on Artificial Intelligence, pp. 138-144, AAAI Press, 2014.

[12] S. Cheng, H. Shen, J. Huang, G. Zhang, and X. Cheng, "StaticGreedy," in Proceedings of the the 22nd ACM international conference, pp. 509-518, San Francisco, California, USA, October 2013.

[13] M. Kimura, K. Saito, and R. Nakano, "Extracting influential nodes for information diffusion on a social network," in Proceedings of the National Conference on Artificial Intelligence, vol. 20, pp. 1371-1376, AAAI Press, 2007.

[14] X. Liu, M. Li, S. Li, S. Peng, X. Liao, and X. Lu, "IMGPU: GPUaccelerated influence maximization in large-scale social networks," IEEE Transactions on Parallel and Distributed Systems, vol. 25, no. 1, pp. 136-145, 2014.

[15] Y. Wang, G. Cong, G. Song, and K. Xie, "Community-based Greedy algorithm for mining top-K influential nodes in mobile social networks," in Proceedings of the 16th ACM SIGKDD International Conference on Knowledge Discovery and Data Mining (KDD '10), pp. 1039-1048, ACM, July 2010.

[16] C. Borgs, M. Brautbar, J. Chayes, and B. Lucier, "Maximizing social influence in nearly optimal time," in Proceedings of the ACM-SIAM, ACM-SIAM, pp. 946-957, 2014.

[17] Y. Tang, X. Xiao, and Y. Shi, "Influence maximization: Nearoptimal time complexity meets practical efficiency," in Proceedings of the 2014 ACM SIGMOD International Conference on Management of Data, SIGMOD 2014, pp. 75-86, USA, June 2014.

[18] H. T. Nguyen, M. T. Thai, and T. N. Dinh, "Stop-and-Stare: Optimal sampling algorithms for viral marketing in billion-scale networks," in Proceedings of the 2016 ACM SIGMOD International Conference on Management of Data, SIGMOD 2016, pp. 695-710, USA, July 2016.

[19] K. Huang, S. Wang, G. Bevilacqua, X. Xiao, and L. V. S. Lakshmanan, "Revisiting the Stop-and-Stare algorithms for influence maximization," in Proceedings of the 43rd International Conference on Very Large Data Bases, VLDB 2017, pp. 913-924, Germany, September 2017.

[20] T. Dinh, H. Nguyen, P. Ghosh, and M. Mayo, "Social influence spectrum with guarantees: computing more in less time," in Computational Social Networks, vol. 9197 of Lecture Notes in Computer Science, pp. 84-103, Springer International Publishing, Cham, 2015.

[21] H. T. Nguyen, T. N. Dinh, and M. T. Thaip, "Cost-aware Targeted Viral Marketing in billion-scale networks," in Proceedings of the IEEE INFOCOM 2016 - IEEE Conference on Computer Communications, pp. 1-9, San Francisco, CA, USA, April 2016.

[22] Y. Liu, D. Pi, and L. Cui, "Mining community-Level influence in microblogging network: A case study on sina weibo," Complexity, vol. 2017, 16 pages, 2017.
[23] J. Li, X. Wang, and K. Deng, "Most influential commuity search over large social networks," ICDE, IEEE, pp. 871-882, 2017.

[24] N. Alduaiji, A. Datta, and J. Li, "Influence propagation model for clique-based community detection in social networks," IEEE Transactions on Computational Social Systems, vol. 5, no. 2, pp. 563-575, 2018.

[25] Y. Li, D. Zhang, and K.-L. Tan, "Real-time targeted influence maximization for online advertisements," in Proceedings of the Vldb Endowment, pp. 1070-1081, Republic of Korea, 2015.

[26] S. Chen, J. Fan, G. Li, J. Feng, K.-L. Tan, and J. Tang, "Online topic-aware influence maximization," in Proceedings of the 41st International Conference on Very Large Data Bases, VLDB 2015, pp. 666-677, USA, September 2015.

[27] X. Wang, Y. Zhang, W. Zhang, and X. Lin, "Efficient DistanceAware Influence Maximization in Geo-Social Networks," IEEE Transactions on Knowledge and Data Engineering, vol. 29, no. 3, pp. 599-612, 2017.

[28] M. Zhong, Q. Zeng, Y. Zhu, J. Li, and T. Qian, "Sample location selection for efficient distance-aware influence maximization in geo-social networks," in Database Systems for Advanced Applications, vol. 10827 of Lecture Notes in Computer Science, pp. 355-371, Springer International Publishing, Cham, 2018.

[29] W. Zhu, W. Peng, L. Chen, K. Zheng, and X. Zhou, "Exploiting viral marketing for location promotion in location-based social networks," ACM Transactions on Knowledge Discovery from Data (TKDD), vol. 11, no. 2, pp. 1-28, 2016.

[30] T. Zhou, J. Cao, B. Liu, S. Xu, Z. Zhu, and J. Luo, "Location-based influence maximization in social networks," in Proceedings of the 24th ACM International on Conference on Information and Knowledge Management, pp. 1211-1220, New York, NY, USA, 2015.

[31] J. Li, T. Sellis, J. S. Culpepper, Z. He, C. Liu, and J. Wang, "Geosocial influence spanning maximization," IEEE Transactions on Knowledge and Data Engineering, vol. 29, no. 8, pp. 1653-1666, 2017.

[32] X. Li, X. Cheng, S. Su, and C. Sun, "Community-based seeds selection algorithm for location aware influence maximization," Neurocomputing, vol. 275, pp. 1601-1613, 2018.

[33] L. Guo, D. Zhang, G. Cong, W. Wu, and K.-L. Tan, "Influence maximization in trajectory databases," IEEE Transactions on Knowledge and Data Engineering, vol. 29, no. 3, pp. 627-641, 2017.

[34] D. Zhang, L. Guo, L. Nie, J. Shao, S. Wu, and H. T. Shen, "Targeted advertising in public transportation systems with quantitative evaluation," ACM Transactions on Information and System Security, vol. 35, no. 3, pp. 1-29, 2017.

[35] J. Li, C. Liu, J. X. Yu, Y. Chen, T. Sellis, and J. S. Culpepper, "Personalized influential topic search via social network summarization," IEEE Transactions on Knowledge and Data Engineering, vol. 28, no. 7, pp. 1820-1834, 2016.

[36] S. Su, X. Li, X. Cheng, and C. Sun, "Location-aware targeted influence maximization in social networks," Journal of the Association for Information Science and Technology, vol. 69, no. 2, pp. 229-241, 2018.

[37] N. Ohsaka, T. Sonobe, S. Fujita, and K.-I. Kawarabayashi, "Coarsening massive influence networks for scalable diffusion analysis," in Proceedings of the 2017 ACM SIGMOD International Conference on Management of Data, SIGMOD 2017, pp. 635650, USA, May 2017.

[38] R. L. Graham, "An efficient algorithm for determining the convex hull of a finite planar set," Information Processing Letters, vol. 1, no. 4, pp. 132-133, 1972. 


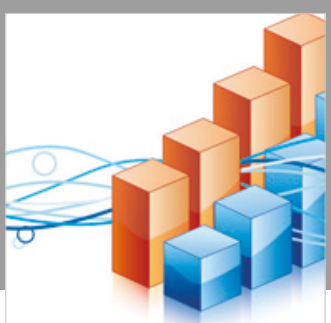

Advances in

Operations Research

\section{-n-m}
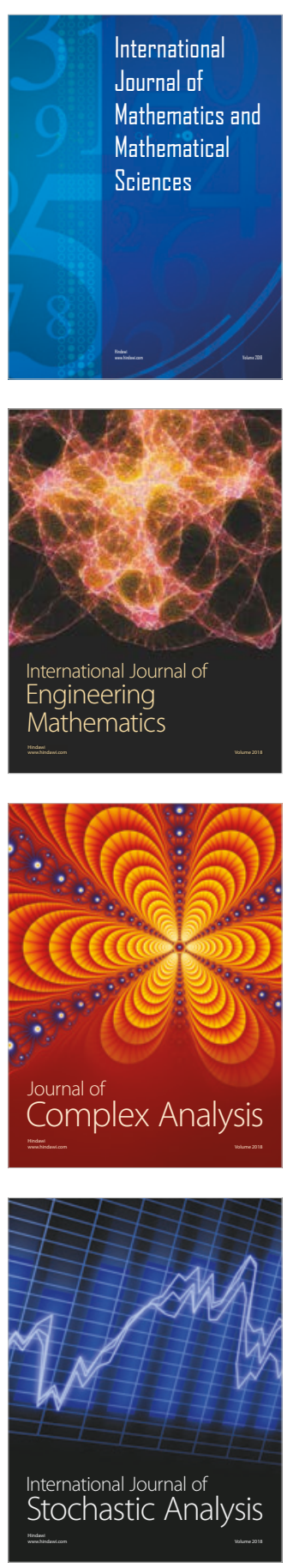
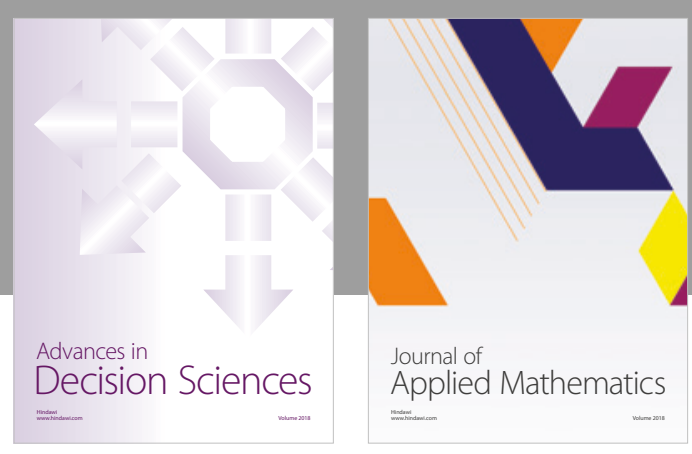

Journal of

Applied Mathematics
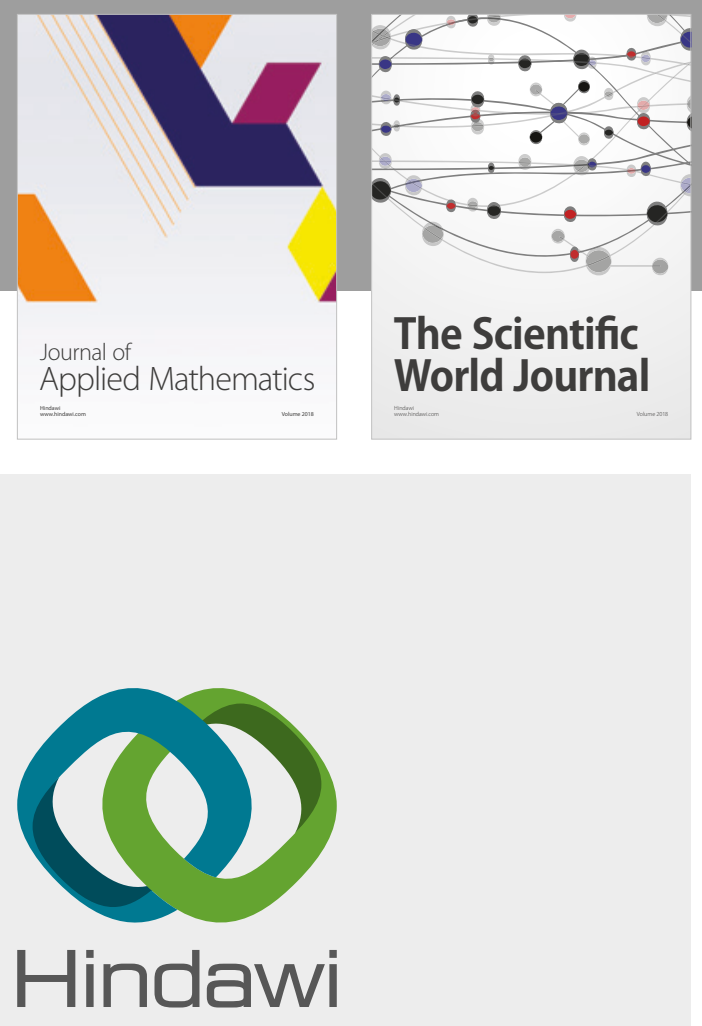

Submit your manuscripts at

www.hindawi.com

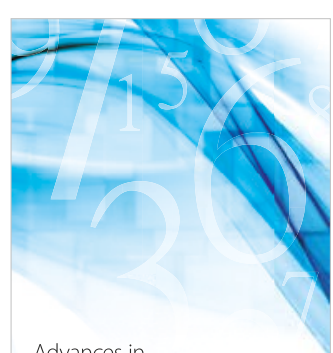

Advances in
Numerical Analysis
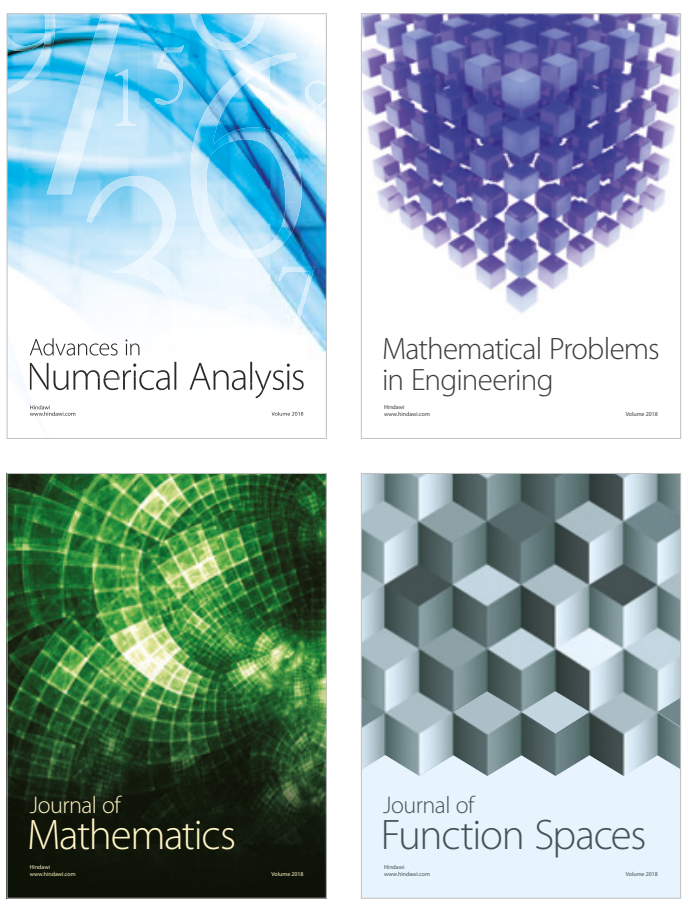

Mathematical Problems in Engineering

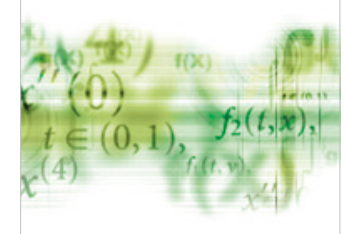

International Journal of

Differential Equations

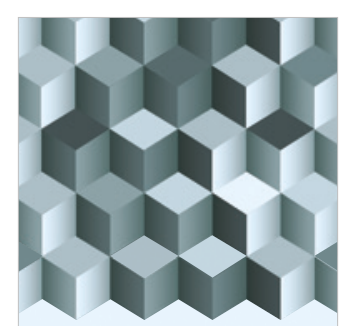

Journal of

Function Spaces
The Scientific

World Journal

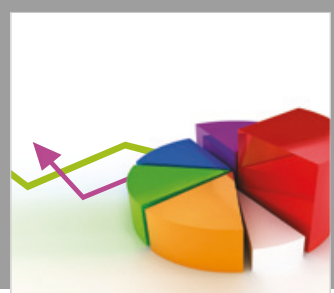

Journal of

Probability and Statistics
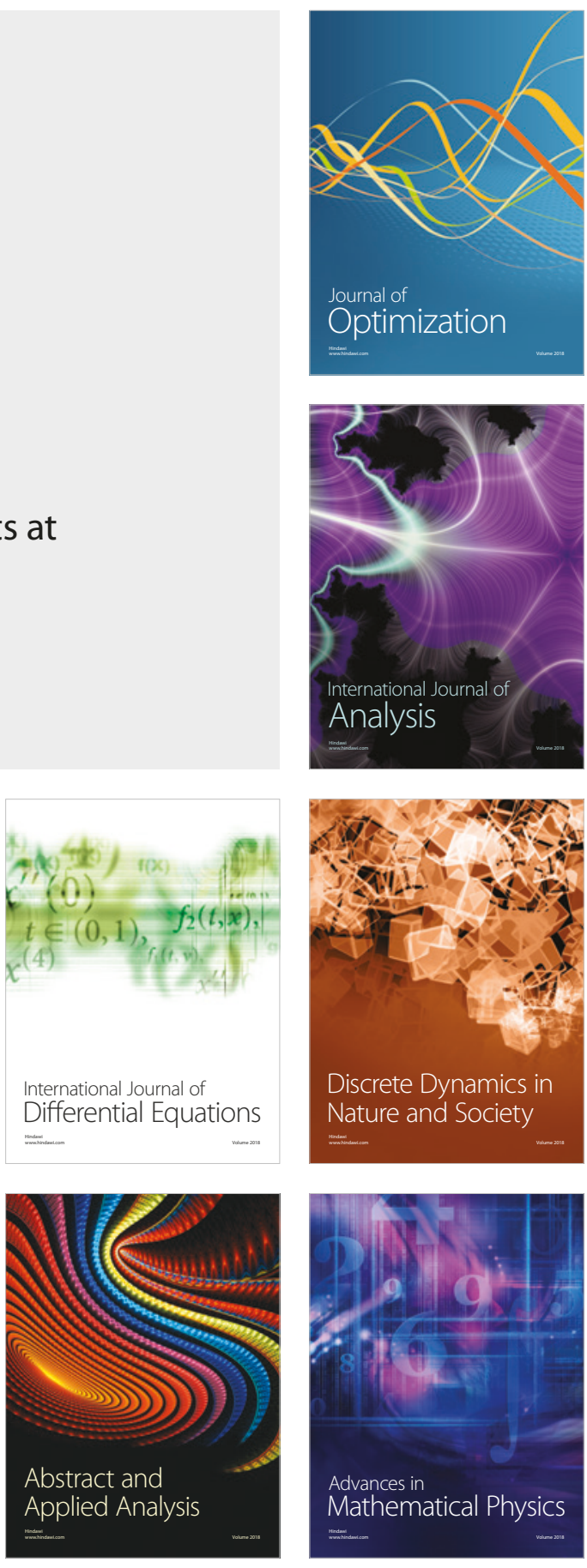